\title{
EGFR NP_005219.2:p.K745_E746insVPVAIK
}

National Cancer Institute

\section{Source}

National Cancer Institute. EGFR NP 005219.2:p.K745 E746insVPVAIK. NCI Thesaurus.

Code C98599.

An insertion of the amino acid sequence valine-proline-valine-alanine-isoleucine-lysine between the lysine at position 745 and the glutamic acid at position 746 of the epidermal growth factor receptor protein. 